\title{
Mineração
}

\section{Estudos experimentais do processo de ressecamento de um rejeito fino de mineração}

\author{
Daviély Rodrigues Silva \\ Samarco Mineração S.A.
}

Waldyr Lopes de Oliveira Filho

Universidade Federal de Ouro Preto

E-mail:waldyr@em.ufop.br

\section{Resumo}

O estudo do ressecamento de um rejeito fino de mineração exposto as condições climáticas naturais e deixado drenar num área-teste é o objeto desse trabalho. $\mathrm{Na}$ área-teste, o material foi depositado sob a forma de lama e a camada formada instrumentada e monitorada durante a fase de ressecamento. A utilização de uma estação meteorológica e as considerações de um modelo hidrológico de evaporação potencial foram muito importantes para a determinação da condição de contorno da superfície. No monitoramento das variáveis do solo, uma técnica considerada de ponta no Brasil foi utilizada para medir diretamente o teor de umidade volumétrica, a técnica do TDR (Time Domain Reflectometry). Os resultados da pesquisa podem ser usados para a determinação de propriedades constitutivas de ressecamento e também na calibração de um modelo numérico para descrição do comportamento do material.

Palavras-chave: Rejeito, ressecamento, investigação de campo.

\begin{abstract}
Desiccation studies of fine mining tailings under natural climatic conditions and resting under a drainage bottom in a test site are presented in this paper. A test section, loaded with several instruments, was implemented in the area to monitor a desiccating layer of the fine tailings especially deposited in the area. A weather station and considerations of a hydrologic model for potential evaporation were very important to characterize the top boundary condition. In monitoring soil variables, an innovative technique was introduced to measure volumetric water content: the time domain reflectometry. Data generated in this research can be used to determine constitutive properties related to the desiccation phenomenon and also be used to calibrated a numerical model to describe soil behavior under desiccation.
\end{abstract}

Keywords: Tailings, desiccation, field investigation. 


\section{Introdução}

Os rejeitos gerados em uma mineração devem ser dispostos de forma segura, tanto tecnicamente, quanto ambientalmente. Entre as opções existentes para disposição de rejeitos finos, destaca-se a do ressecamento, isto é, a exposição do material às condições climáticas e de drenagem.

Para investigar as condições de contorno e as do material envolvidas no processo de ressecamento para um rejeito particular, foi construída uma áreateste na barragem de rejeitos do Germano da Samarco Mineração S.A., em Mariana (MG). Essa área foi preenchida com uma camada do material, teve uma seção instrumentada com diversos equipamentos e nela foi realizado um acompanhamento do comportamento do material durante todo período de teste.

Para se fazer um levantamento das informações necessárias para descrever o processo de ressecamento, várias técnicas e procedimentos foram utilizados, entre os quais destaca-se o uso de sondas TDR (Time Domain Reflectometry) ainda pouco conhecido no País.

\section{Fenômeno do ressecamento}

O fenômeno do ressecamento é típico de solos finos e consiste na contração do solo devido à perda d'água por meio da evaporação (superfície) e/ou drenagem (base), sendo a sucção gerada enquanto o solo resseca o principal responsável pelo mecanismo.

Esse processo ocorre em dois estágios: unidimensional, quando ocorre apenas contração vertical (recalque); e tridimensional, quando surgem descontinuidades no solo, chamadas trincas de ressecamento, e a redução de volume é resultado tanto de recalques quanto das contrações laterais livres. A inabilidade do solo em não suportar as tensões de tração existentes nessa fase é a responsável pela abertura das trincas. Para o estudo desse fenômeno, é necessário conhecer as propriedades do solo e as condições de contorno que o envolvem.
A perda d'água do solo por evaporação é uma condição de contorno importante para a evolução do fenômeno e ela pode ocorrer em dois estágios sucessivos para um solo que resseca partindo de uma condição saturada: (a) quando é controlada pelas condições meteorológicas e (b) quando é controlada pelas propriedades de fluxo do solo e, em especial, o nível de sucção. Além da radiação solar, outras variáveis exercem influência no processo de evaporação, tais como: a ação do vento, temperaturas da água e do ar e a umidade relativa do ar.

A drenagem é outra condição de contorno que pode concorrer no processo de ressecamento. A drenagem corresponde ao processo de se criar uma fronteira onde a água possa ser removida por processo de fluxo gravitacional ou induzido.

Devido à evaporação e/ou drenagem e a conseqüente contração do solo, surgem as trincas. A morfologia dessas descontinuidades é dependente da mineralogia do solo e das condições climáticas. As primeiras trincas formadas são chamadas primárias, com orientação paralela, e depois surgem as secundárias, que, por sua vez, são aproximadamente normais às primárias, e o conjunto forma colunas de solo com seções quadrangulares ou hexagonais na superfície.

A contração do solo devido à perda de água (shrinkage) é um dos fenômenos importantes do solo e ocorre em solos coesivos. Os estágios em que esse processo ocorre são: normal, residual e zero (Oliveira Filho, 1998). Na fase de contração normal, a redução de volume é igual à perda de água. Na fase residual, a redução volumétrica é menor do que o volume de água perdido, surgindo ar nos vazios. A fase de contração zero ocorre quando as partículas do solo atingem sua configuração mais densa e não há mais redução volumétrica. O solo se encontra em condição saturada até o início da fase residual, próximo do limite de contração de Atterberg.

A seguir, é apresentada a equação governadora do processo de ressecamento proposta por Abu-Hejleh e Znidarcic (1995):

$\frac{\partial}{\partial a 1}\left[K-\frac{K}{\gamma_{w}} \frac{e \gamma_{w}+\gamma_{s}}{1+e}\left(1-\frac{\partial \sigma^{\prime}{ }_{v}}{\partial e_{c r}} \frac{d e_{c r}}{d \sigma_{v}}\right)-\frac{K}{\gamma_{w}} \frac{\alpha\left(1+e_{c r}\right)}{1+e}\left(\frac{\partial \sigma^{\prime}{ }_{v}}{\partial e} \frac{\partial e}{\partial a 1}\right)\right]-\eta v \frac{\partial \alpha}{\partial a 1}=\frac{1}{\alpha\left(1+e_{c r}\right)} \frac{\partial e}{\partial t}$

onde: $e=$ índice de vazios; $e_{c r}=$ índice de vazios de abertura de trinca; $\sigma^{\prime}{ }_{v}=$ tensão efetiva vertical; $\sigma_{v}=$ tensão total vertical; $\gamma_{w}=$ peso específico da água; $\gamma_{s}=$ peso específico dos grãos; $k=$ função condutividade hidráulica; $a_{1}=$ coordenada espacial lagrangiana; $v=$ taxa de evaporação; e $t=$ tempo.

Para a solução dessa equação, é necessário o conhecimento de algumas funções constitutivas: relações de compressibilidade e permeabilidade sob condições de contração unidimensional e tridimensional $\left(e \times \sigma^{\prime}, e \times k\right)$; propriedade constitutiva relativa ao início e propagação das trincas $\left(e_{c r} \times \sigma_{v}\right)$; um funcional, " $\alpha$ ", que associa a deformação vertical e lateral durante a contração tridimensional e; um

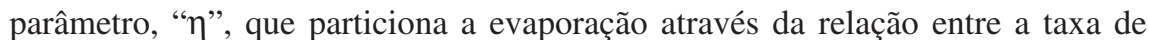
evaporação nas paredes das trincas e a evaporação na superfície. Para todas essas propriedades do material, existem técnicas de ensaio específicas também descritas pelos autores (Abu-Hejleh \& Znidarcic, 1995).

\section{Metodologia para o estudo}

O material em estudo é um dos rejeitos da Samarco que se caracteriza por ser um material fino (abaixo de $74 \mu \mathrm{m}$ ). Esse rejeito foi lançado através de bombeamento para uma área-teste construída com a finalidade de estudar o comportamento desse material. Montaram-se, nessa área, uma passarela, para que se tivesse acesso ao centro da mesma, e um vertedouro, para que a água superficial pudesse ser retirada. 
No laboratório, foi realizado o ensaio de contração livre, para se determinarem o limite de contração (LC) e o funcional " $\alpha$ ", requerido na teoria de ressecamento. Nesse ensaio, parte-se de uma amostra na condição saturada, próximo do limite de liquidez (LL), e se permite ao solo perder água por evaporação, controlando-se a redução de volume total e determinando-se o teor de umidade. A curva de contração é muito importante, pois permite determinar-se o LC e se ter uma boa estimativa até que teor de umidade do solo permaneça saturado.

Para se conhecerem o comportamento das variáveis do solo e as variáveis relacionadas às condições de contorno, instrumentos de diversos tipos foram instalados na área teste. Além disso, várias amostragens foram realizadas durante o período de estudos.

Utilizou-se uma estação meteorológica para determinar as condições de contorno climáticas. A estação fabricada pela Davis Instruments ${ }^{\odot}$ contém um conjunto de sensores capaz de obter dados sobre a chuva, temperatura, umidade relativa, velocidade e direção do vento e radiação solar. Os dados eram registrados em um "datalogger" e descarregados periodicamente em um "laptop", gerando relatórios e gráficos. Os dados de evaporação eram estimados pela estação através da utilização de um modelo hidrológico (Penman modificado), citado em Snyder (1992).

Os sensores do solo utilizados foram os de umidade (TDR), de temperatura e de sucção. O TDR (Time Domain Reflectometry) mede o teor de umidade volumétrica do solo a partir do princípio de propagação das ondas eletromagnéticas de acordo com a variação da constante dielétrica. O sensor de sucção utilizado foi de bloco poroso de gesso do tipo elétrico. Ele indica com razoável acurácia o déficit de água para os valores compreendidos entre 0 e $1000 \mathrm{kPa}$. O sensor de temperatura mede a temperatura do solo através de um termistor e opera na faixa de $-50^{\circ}$ a $+95^{\circ} \mathrm{C}$. Todos esses sensores são da Campbell Scientific ${ }^{\odot}$ inclusive o "datalogger" CR10X utilizado para registrar e coletar os dados dos mesmos.

Para a instalação dos instrumentos, foi realizada uma análise numérica preliminar utilizando o programa CONDESO (Yao \& Znidarcic 1997) sobre ressecamento, levando-se em consideração as condições de contorno existentes e as características do solo.

Para se ter a medida do recalque, instalaram-se uma régua de $1,5 \mathrm{~m}$ no centro da área-teste e uma trena de 2,0 m em um dos apoios da passarela. O nível de água na camada de lama e na base foi determinado a partir da instalação de dois piezômetros do tipo Casagrande na base de rejeito arenoso. Foram instalados, também, três tensiômetros de mercúrio, um na base e dois em diferentes alturas na camada de lama, para se obterem informações de sucção.

Ainda com a área-teste vazia, foram instalados a estação meteorológica, a régua e a trena, os dois piezômetros e o tensiômetro da base como mostra a Figura 1. Conjuntos de sensores formados por medidores de sucção, temperatura e TDR foram lançados na área-teste à medida que acontecia o seu enchimento. $\mathrm{O}$ primeiro conjunto foi lançado quando a altura da camada de lama era $57,4 \mathrm{~cm}$. O

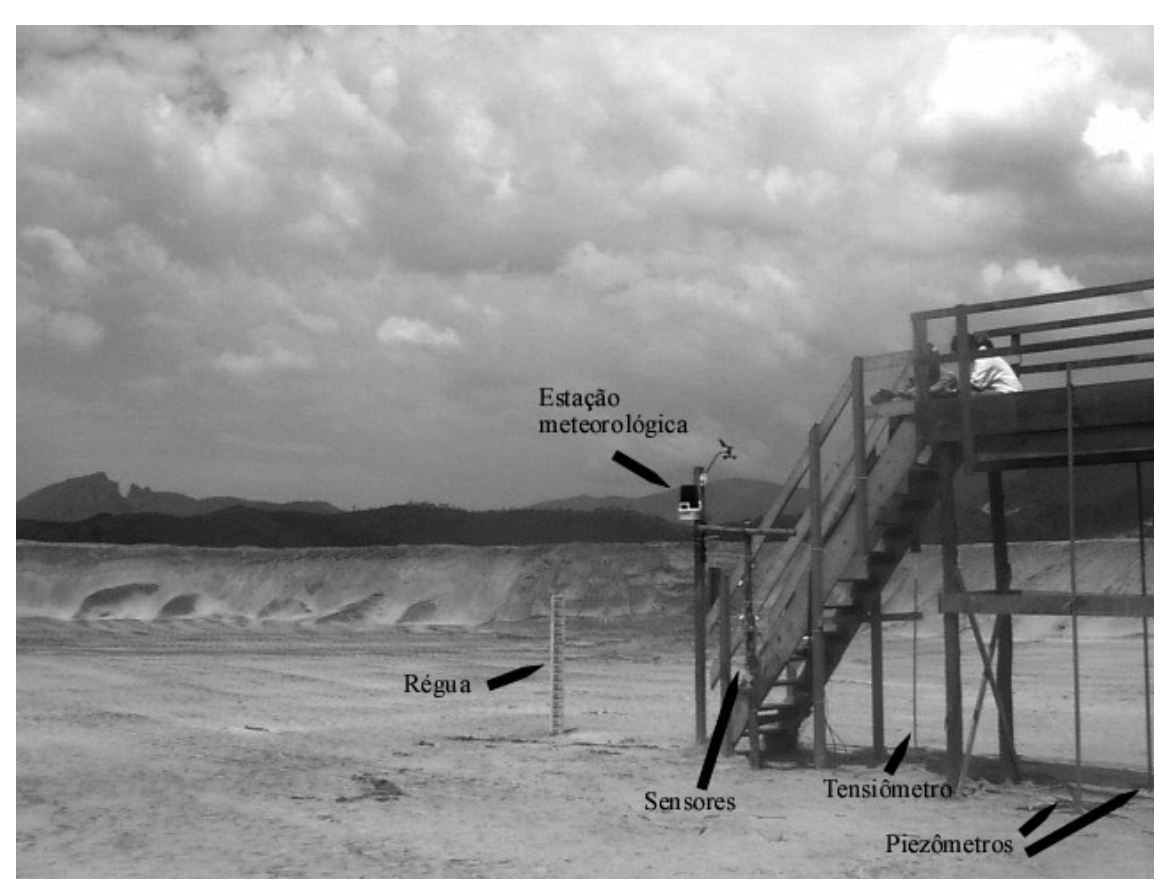

Figura 1 - Vista geral da área-teste antes do enchimento. segundo lançou-se a uma altura de 70 $\mathrm{cm}$ e o terceiro, com $81 \mathrm{~cm}$. Finalmente, quando se atingiu a altura de $106 \mathrm{~cm}$, o último conjunto foi liberado. $\mathrm{O}$ enchimento da área foi realizado até a altura de $134 \mathrm{~cm}$.

Para que o processo de secagem se inicie, é necessário que não haja lâmina d'água sobre o material. Logo, toda água foi drenada através do vertedouro e, assim, permitiu-se o início do ressecamento da lama.

Foram realizadas coletas de amostras na fase onde a lama estava mais líquida a partir de um amostrador de caneco e na fase mais consistente através do amostrador de pistão. Com o aumento da consistência da lama, foi possível coletar apenas duas amostras na primeira fase. As primeiras amostras com o pistão foram coletadas após um dia de ressecamento e, durante o tempo de estudo, foram realizadas 21 amostragens em pontos diferentes, abrangendo a área próxima dos sensores. As amostras, depois de extrudadas e feitas as medidas para o cálculo da densidade, eram levadas em sacos plásticos para o laboratório, onde procedia-se o ensaio de umidade gravimétrica e, para algumas, procedia-se a determinação da densidade real dos grãos. 


\section{Resultados}

\subsection{Contração}

Os resultados do ensaio de contração livre permitiram determinar a curva de contração mostrada na Figura 2. A análise do gráfico permite que se estime o Limite de Contração em torno de $28 \%$, sugerindo, também, que até essa umidade o solo estaria saturado.

\subsection{Recalque}

A variação da altura da camada (recalques) é mostrada na Figura 3, com destaque para a etapa de ressecamento. Nesse período, partiu-se de uma altura da camada de lama de $108 \mathrm{~cm}$ e após 2 meses tinha-se uma altura de $92 \mathrm{~cm}$. Logo, em um período de 61 dias, obteve-se um recalque vertical de $16 \mathrm{~cm}$. Observou-se uma diminuição contínua da altura da camada indicando uma irreversibilidade do processo mesmo considerando-se que houve períodos de chuvas.

Nota-se, no entanto, que a taxa de recalque não é constante, podendo-se identificar duas pendentes distintas na curva de recalque, sendo o ponto de inflexão por volta do dia 115 .

\subsection{Umidade volumétrica}

A evolução do perfil de umidade volumétrica $(\theta)$ com o tempo obtido com a técnica do TDR é mostrada na Figura 4. No geral, a tendência do teor de umidade volumétrica foi diminuir com o tempo no período de ressecamento. Enquanto havia uma camada superficial de água na área (período anterior ao ressecamento), pode-se considerar que a umidade não teve variações representativas. Com o início do ressecamento, notase que o topo da camada teve imediatamente uma redução da

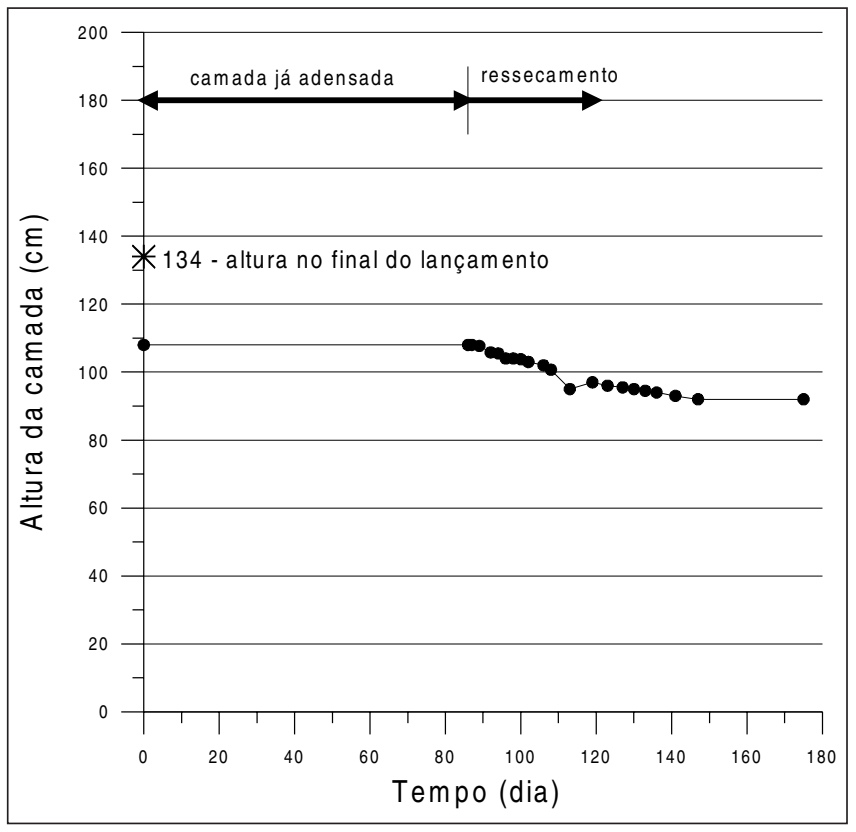

Figura 3 - Variação da altura da camada (recalques) no tempo. umidade, enquanto as outras elevações ainda levaram um certo tempo para que essa diminuição fosse percebida. Além disso, os dados iniciais mostram uma certa variação de $\theta$, ao passo que, no final, percebe-se uma homogeneização nos resultados.

\subsection{Teor de sólidos}

Uma grandeza derivada muito importante para análise e aplicações práticas é o teor de sólidos, P. Ele pode ser calculado a partir de teor de umidade e da densidade real dos grãos obtidos com as amostras coletadas durante o ressecamanto.

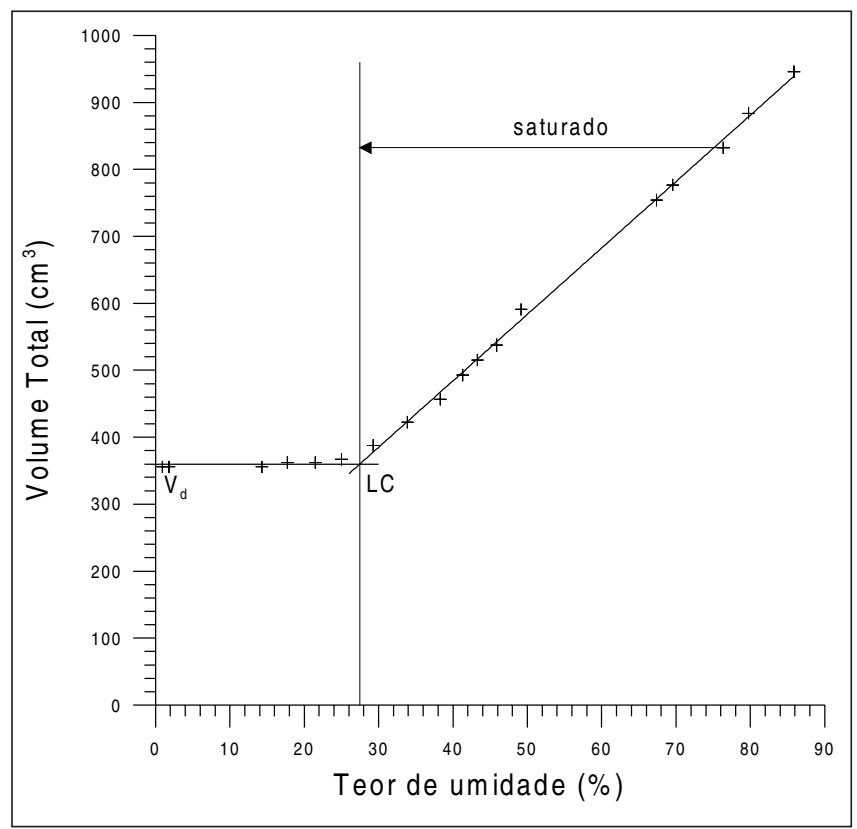

Figura 2 - Resultados do ensaio de contração livre.

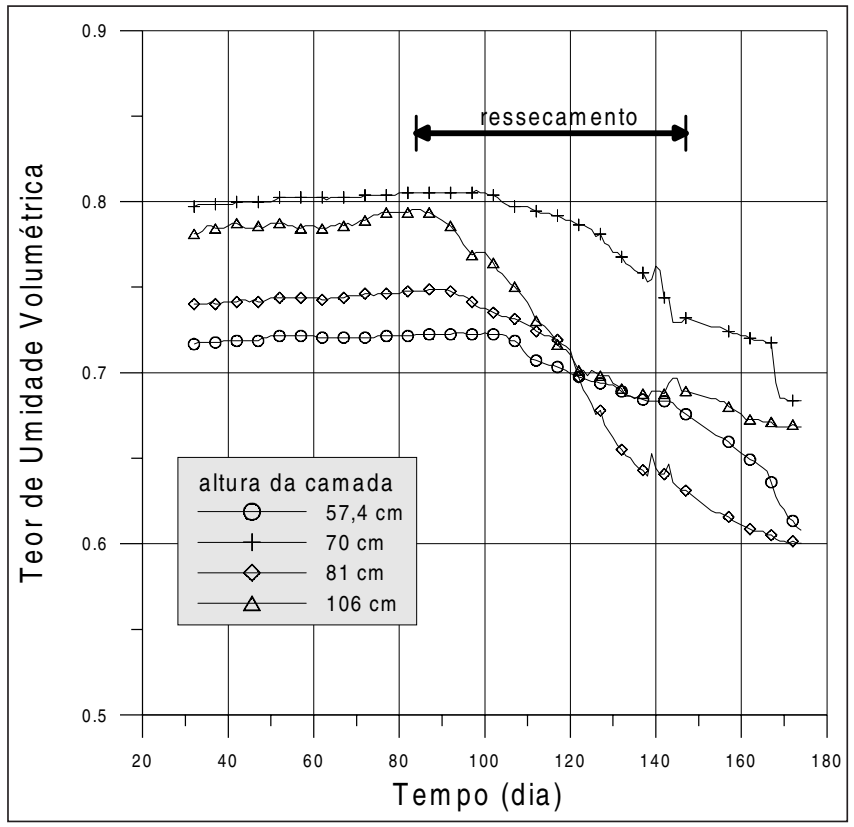

Figura 4 - Evolução do teor de umidade volumétrica no tempo. 
Como pode ser observado na Figura 5, o perfil de teor de sólidos evoluiu de forma expressiva durante o período. Constata-se um ganho médio dessa variável da ordem de $30 \%$. Se considerarmos os valores de lançamento $(\mathrm{P}=20 \%)$, esse ganho é muitíssimo maior.

Observa-se, ainda, que a variação do teor de sólidos é maior quanto mais próximo da superfície. Há uma tendência também do teor de sólidos ser maior na base. Esses valores maiores de $P$ na base refletem bem sua condição drenante.

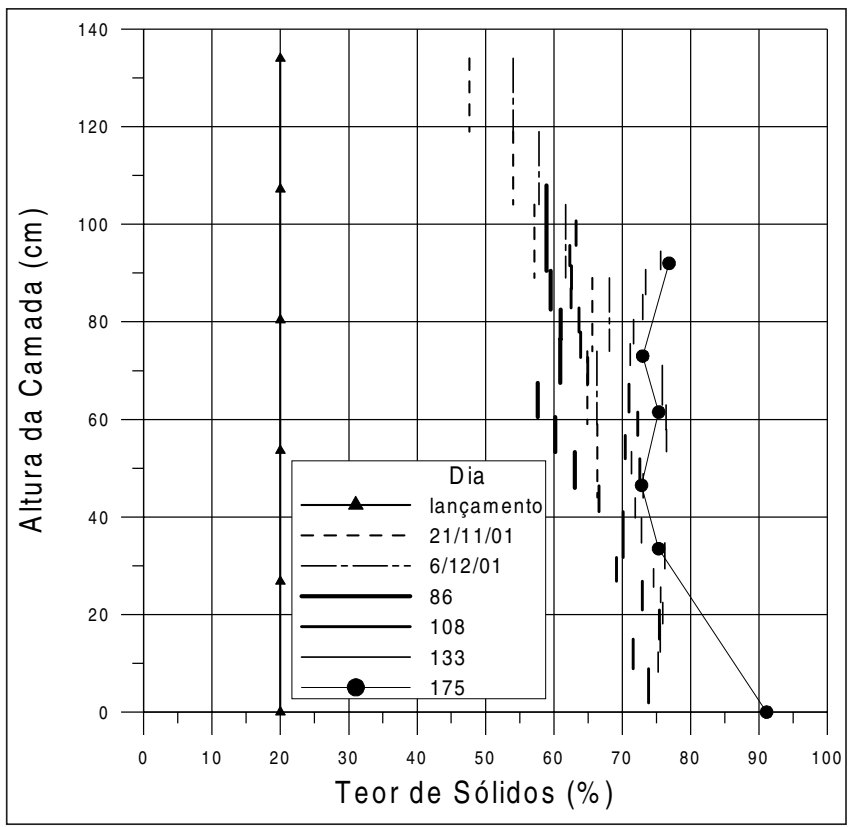

Figura 5 - Perfis de teor de sólidos para diversos tempos.

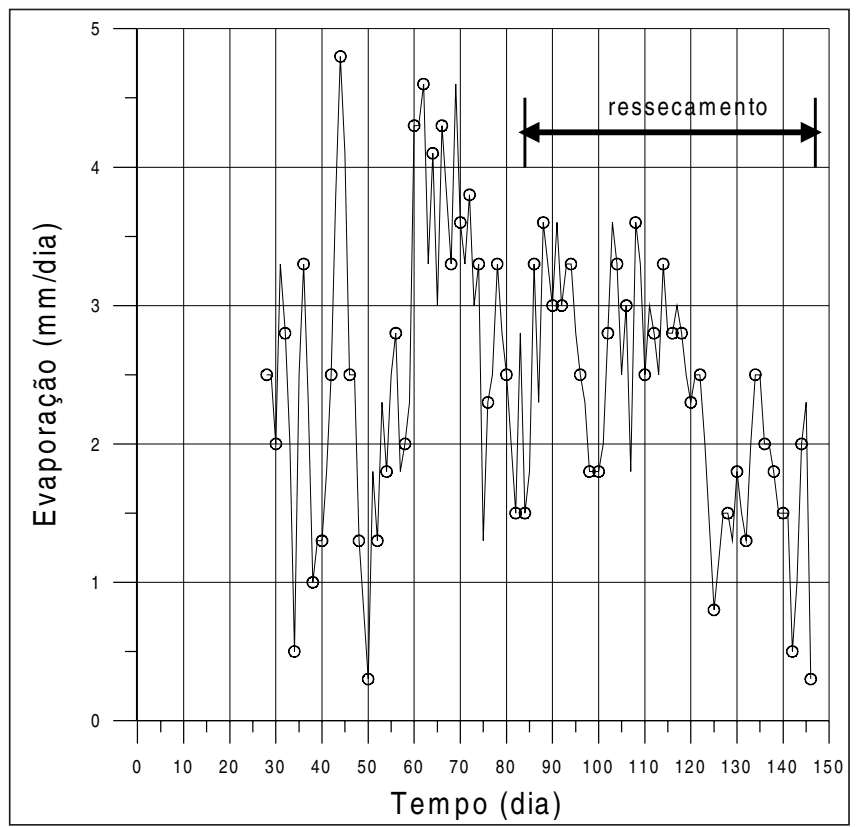

Figura 6 - Evaporação potencial medida pela estação meteorológica.

\subsection{Evaporação}

Analisando os dados de evaporação na Figura 6, podese constatar duas médias. Do início do ressecamento (dia 84) até o dia 120, aproximadamente, a taxa média estava em torno de $2.8 \mathrm{~mm} /$ dia, caindo para algo em torno de $1,5 \mathrm{~mm} /$ dia no final do período. Essas taxas diferentes coincidem com a mudança na evolução dos recalques, também mostrado na Figura 2, que acontece precisamente entre os dias 115 e 120, passando de uma pendente maior para outra menor.

\section{Conclusão}

Os resultados dessa pesquisa mostram que a metodologia utilizada foi eficiente na obtenção das informações necessárias à descrição do fenômeno do ressecamento. Essa metodologia teve como base uma teoria sobre o ressecamento de solos finos de reconhecida qualidade e aceitação (Abu-Hejleh e Znidarcic, 1995). Dessa forma, foi possível que todas as variáveis medidas tivessem suas grandezas estimadas, não só em termos qualitativos, mas também quantitativos. Dentro da metodologia utilizada, houve sempre uma preocupação com a consistência dos resultados.

O ensaio de contração livre permitiu que se conhecesse o teor de umidade correspondente ao limite de contração do solo e que também se conhecesse até onde o solo se comporta no estado saturado.

A instrumentação desse trabalho foi extensa e variada e, em alguns casos, até inovadora (sonda TDR), não se tendo notícia no Brasil de estudos sobre o fenômeno tão documentados.

Outro aspecto de importância no trabalho foi o tratamento da interação solo e atmosfera em profundidade, combinando aspectos iminentemente geotécnicos com os hidrológicos, estes últimos referentes à evaporação.

\section{Agradecimentos}

Agradecemos a SAMARCO Mineração S.A. pelo apoio.

\section{Referências bibliográficas}

ABU-HEJLEH, A.N., ZNIDARCIC, D. Desiccation theory for soft cohesive soils. Journal of Geotechnical Engineering, ASCE, v. 121, n. 6, p. 493-502. 1995.

OLIVEIRA FILHO, W.L. Verification of a desiccation theory for soft soils. Boulder: Department of Civil, Environmental and Architectural Engineering, University of Colorado, 1998. (Tese de Doutorado).

SNYDER, R.L. E PRUITT, W.O. Evapotranspiration data management in California. In: Irrigation \& Drainage, Session Proceedings/Water Forum'92, EE.HY,IR.WR Div/ASCE, Baltimore, MD/August 2-6. 1992.

WILSON, G.W., FREDLUN, D.G., BARBOUR, S.L. The Effect of soil Suction on Evaporative Fluxes from Soil Surfaces. Canadian Geotechnical Journal, v. 34, p. 145-155. 1997.

YAO, D.T.C., ZNIDARCIC, D. User's manual for computer program CONDES. Boulder: Department of Civil, Environmental and Architectural Engineering, University of Colorado, 1997.

Artigo recebido em 21/03/2003 e aprovado em 19/12/2003. 